\title{
FREQUENCY OF ACUTE KIDNEY INJURY AMONG PATIENTS UNDERGOING CORONARY ANGIOGRAPHY
}

\author{
Sajid Khan, Abdul Hameed Siddiqui, Ariz Samin, Syed Hassan Mustafa*, Akhtar Gul, Muhammad Waqas Mazhar, \\ Farhan Tuyyub, Naseer Ahmed Samore
}

Armed Forces Institute of Cardiology/National Institute of Heart Disease (AFIC/NIHD)/National University of Medical Sciences (NUMS) Rawalpindi Pakistan, *Ayub Teaching Hospital Abbottabad Pakistan

\begin{abstract}
Objective: To determine the frequency of acute kidney injury among patients undergoing coronary angiography. Study Design: Descriptive cross-sectional study.

Place and Duration of Study: Department of Cardiology, Hayatabad Medical Complex, Peshawar, from Jan 2018 to Jul 2018.

Methodology: This study was conducted in the in the Department of Cardiology, Hayatabad Medical Complex, Peshawar from 22 nd Jan 2018 to 22nd Jul 2018. Through a descriptive cross-sectional study design, a total of 116 patients scheduled for coronary angiography were included in the study in a consecutive manner and baseline / follow up serum creatinine was recorded to detect acute kidney injury.

Results: In this study 116 patients were included, $61.2 \%$ males and 38.8\% females. Mean age of the patients was 55.6 years with a standard deviation of 6.6 years. Mean baseline serum creatinine level was $0.9 \pm 0.11 \mathrm{mg} / \mathrm{dl}$ which was $1.5 \pm 0.1148$ hours after coronary angiography $(p 0.000)$. AKI was recorded in $19.8 \%$ of patients.

Conclusion: Acute kidney injury after coronary angiography is not uncommon in our population. More studies are recommended on its risk factors and complications to draw future directions for its control and prevention.
\end{abstract}

Keywords: Acute kidney injury, Contrast induced nephropathy, Creatinine, Myocardial infarction.

This is an Open Access article distributed under the terms of the Creative Commons Attribution License (http://creativecommons.org/licenses/by/4.0), which permits unrestricted use, distribution, and reproduction in any medium, provided the original work is properly cited.

\section{INTRODUCTION}

Large doses of iodinated contrast media (CM) are often indispensable for percutaneous cardiac interventions. Most of the contrast media are usually harmless however they can cause contrast induced nephropathy. Since worldwide the number of coronary angiographies performed continues to rise, as does the contrast induced nephropathy or contrast induced acute kidney injury (CIAKI). CIAKI in developed countries have become the third leading cause for acute renal failure ${ }^{1}$. Patients with CIAKI may suffer from long term morbidity which may include progression to chronic renal failure, and has up to $40 \%$ inhospital mortality ${ }^{2,3}$.

Patients with preexisting comorbidities, including those with diabetes mellitus, chronic kidney disease (CKD), and heart failure, are at particularly high risk of CIAKI ${ }^{4}$. The primary manifestation is a drop in renal function that

Correspondence: Dr Sajid Khan, Department of Interventional Cardiology, AFIC/NIHD Rawalpindi Pakistan occursa few days after exposure to the contrast media ${ }^{6}$. The declining renal functions however stops and return to normal baseline levels a week or 2 after expose and usually does not requires renal replacement therapy ${ }^{5,6}$.

The frequency of contrast induced acute kidney injury after coronary angiography / percutaneous coronary intervention is about $15 \%^{7}$ and which may also include coronary angiography performed before bypass surgeries and/ or valve replacement surgeries. Since heart surgery is associated with the risk of post-operative AKI, concerns have been raised as to the combined risk of angiography and surgery when performed in close succession ${ }^{8}$. There has been contradicting research on this concern with some advocating the risk and others recommending that cardiac surgery be performed 5 days after exposure to contrast media9 .

Literature shows conflicting results regarding whether or not CIAKI progress into Chronic Renal Failure (CRF). While study shows that 
CIAKI is significantly associated with progressing into chronic renal failure ${ }^{10,11}$, others have advocated that there does not exist any such association $^{6}$.

In Neyra et al, a sample of 1160 patients were studied for the development of CIAKI and it was found that it occurred in 19\% of CRF cases and in $18 \%$ of non-CRF cases ${ }^{12}$. The other reported incidence of CIAKI after coronary intervention is $23 \%^{13}$ and $29.5 \% 14$.

The present study is designed to determine the frequency of CIAKI after coronary angiography. As mentioned above, the incidence of AKI after coronary interventions is being reported different at different centers and also the existing data failed to associate the occurrence of AKI after coronary interventions. Even if it occurs, the morbidity and mortality is high among patients who develop AKI. In this connection, the present study highlighted the magnitude of AKI after coronary angiography. The results of this study gave a fresh local first hand evidence for cardiologists to brainstorm about the future of coronary angiography in light of preventing AKI.

\section{METHODOLOGY}

This study was conducted in the in the Department of Cardiology, Hayatabad Medical Complex, Peshawar from 22nd Jan 2018 to 22nd Jul 2018. A Sample of 116 was included through non probability consecutive sampling, with sample size being calculated using $18 \%^{12}$ proportion of AKI after coronary angiography, 95\% confidence interval and 7\% margin of error. Our sample included patients 18 years of age or above with baseline creatinine below $1.2 \mathrm{mg} / \mathrm{dl}$ who were undergoing coronary angiography for any reason. Patients with prior history of congestive chronic heart failure, acute or chronic kidney failure, chronic liver disease or renal transplant were excluded from the study. Acute kidney injury was defined as any rise in creatinine more than 26 micromole/liter in $48 \mathrm{hrs}$ or more than 1.5 times the baseline.

The study was conducted after approval from hospitals ethical and research committee.
All patients included in the study and who have undergone percutaneous coronary angiography was followed up for $48 \mathrm{hrs}$ post angiography and $3 \mathrm{ml}$ blood was sent to the hospital laboratory to check serum level of creatinine. AKI was confirmed if rise in serum creatinine level is more than 26 micromole/litre OR more than 1.5 times of the baseline. Data was stored in preformed proforma.

Data collected was analyzed using SPSS version 20. Frequency and percentage was calculated for categorical variables like gender and AKI while Mean \pm SD was calculated for numerical variable like age, baseline creatinine \& follow up creatinine. AKI was stratified among age and gender to see the effect modifications. All results were presented in the form of tables and graphs.

\section{RESULTS}

The study was conducted on 116 scheduled for coronary angiography. The mean age of our sample was 55.6 years with a standard deviation of 6.6 years. We divided the patients in 3 different age groups and we found that in the age group up to 50 years we had 31 patients $(26.7 \%)$, in the

Table-I: Baseline characteristics of patients.

\begin{tabular}{l|l|c}
\hline \multicolumn{2}{l|}{ Variables } & $\mathbf{n = 1 1 6}$ \\
\hline Age (Mean \pm SD) & $55.63 \pm 6.62$ years \\
\hline \multirow{2}{*}{ Gender } & Males & $71(61.2 \%)$ \\
\cline { 2 - 3 } & Females & $45(38.8 \%)$ \\
\hline \multirow{2}{*}{$\begin{array}{l}\text { Serum } \\
\text { Creatinine }\end{array}$} & $\begin{array}{l}\text { Baseline Serum } \\
\text { Creatinine }\end{array}$ & $0.9 \pm 0.12 \mathrm{mg} / \mathrm{dl}$ \\
\cline { 2 - 3 } & $\begin{array}{l}\text { Follow Up } \\
\text { Creatinine }\end{array}$ & $1.5 \pm 0.10 \mathrm{mg} / \mathrm{dl}$ \\
\hline \multicolumn{2}{l}{ Acute Kidney Injury } & $23(19.8 \%)$ \\
\hline
\end{tabular}

Table-II: Age groups wise stratification of AKI (n=116).

\begin{tabular}{l|c|c}
\hline Age Group & \multicolumn{1}{|c|}{ Acute Kidney Injury } & \multirow{2}{*}{$p$-value } \\
\hline Upto 50 years & - & \multirow{2}{*}{0.001} \\
\hline $50-60$ years & $7(11.3 \%)$ & $16(69.6 \%)$ \\
\hline$>60$ years & wise stratification of AKI \\
\hline $\begin{array}{l}\text { Table-III: Gender } \\
\text { (n=116). }\end{array}$ & Acute Kidney Injury & $p$-value \\
\hline Gender & $16(22.5 \%)$ & 0.358 \\
\hline Male & $7(15.6 \%)$ & \\
\hline Female &
\end{tabular}

age group 50 to 60 years we had $62(53.4 \%)$ and in the group 60.01 years and above we had 
$23(19.8 \%)$ patients. The youngest patient was 43 years while the oldest was 65 with mean and $\mathrm{SD}$ as 55.63 and 6.62 respectively. There were 71 (61.2\%) males and 45 (38.8\%) females.

Mean baseline serum creatinine level was 0.9 $\pm 0.12 \mathrm{mg} / \mathrm{dl}$ which was $1.5 \pm 0.1048$ hours after coronary angiography $(p-0.000)$. AKI was recorded in $23(19.8 \%)$ of patients. Results are shown in tables.

\section{DISCUSSION}

Acute kidney injury (AKI) after coronary angiography is often attributed to radio contrastassociated kidney injury ${ }^{15}$ and is now a common cause of AKI in hospitalized patients ${ }^{1}$. Patients with pre-existing co morbidities, including those with diabetes mellitus, chronic kidney disease (CKD), and heart failure, are at particularly high risk of contrast-induced AKI (CI-AKI) ${ }^{4}$. The primary manifestation is a drop in renal function that occurs a few days after exposure to the contrast media 5 . The declining renal functions however stops and return to normal baseline levels a week or 2 after expose and usually does not requires renal replacement therapy ${ }^{5}$.

Several observational studies suggest that this drop in renal function after contrast media exposure are associated with increased morbidity, including longer hospital admission, higher risk of coronay artery diseases and increased mortality ${ }^{2,15}$. Although some of these findings have been summarized in narrative reviews 14,16 , the interpretation of these findings has remained controversial given the correlation between preexisting clinical variables that are associated with both CI-AKI and adverse clinical outcomes, variability in adjustment for potential confounders across observational studies, and uncertainty whether reported risks are indeed attributable to CI-AKI.

The association between even small changes in serum creatinine and adverse short-term clinical outcomes has been documented repeatedly ${ }^{17,18}$. Graded increases in mortality within 30 days of coronary angiography and increased length of hospital admission have been shown to correlate with increases in the severity of AKI after coronary angiography ${ }^{15}$. Among patients receiving percutaneous coronary interventions, AKI has been shown to be associated with other early complications, including MI, target vessel reocclusion, postprocedural bleeding complications ${ }^{19}$, and the need for mechanical ventilation or circulatory support ${ }^{17,19}$.

CIAKI has been found to have long term implications in the form of future cardiovascular or renal event ${ }^{20}$. These long term complications after AKI have several possible explanations. First, patients who develop AKI have a higher prevalence of comorbidities such as diabetes mellitus, heart failure, and chronic kidney disease, each of which may increase the risk of heart failure, progression to kidney failure, and death ${ }^{21}$. Second, AKI may identify patients with impaired cardiac output or renal hemodynamic vulnerability who are at heightened long-term risks for decompensated heart function, loss of kidney function, and death. The long-term risks of adverse outcomes after AKI may be related to longterm effects on kidney function after an episode of AKI. Recent studies suggest that episodes of AKI contribute to persistent loss of kidney function, faster subsequent rate of decline in kidney function ${ }^{22}$, processes that have been associated with future risks of episodes of heart failure and progression to ESRD ${ }^{23}$. Regardless of causality, the occurrence of AKI does appear to accurately identify a group of patients at higher risk for these adverse events, suggesting that targeting these patients for careful outpatient management has the potential to improve long-term outcomes.

These findings are important because a number of therapeutic interventions have been shown to be of value in improving survival, slowing the progression to ESRD, and preventing hospital admissions in general populations of patients with chronic kidney disease or heart failure ${ }^{24}$. Early clinical follow-up, evaluation of volume status, use of diuretics, and inhibitors of the renin-angiotensin system have the potential to improve these outcomes after an episode of AKI; however, further research is needed to evaluate 
the role of these therapies specifically in survivors of AKI after coronary angiography.

Neyra et al ${ }^{11}$ studied a sample of 1160 patients and observed that contrast induced nephropathy occurred in $19 \%$ of CRF cases and in $18 \%$ of non-CRFpatients. They also observed that patients who required assisted ventilation and those who needed ionotropes were at an increased risk of developing contrast induced nephropathy and such patients had higher morbidity and mortality. Another study also showed that both CKD patients and non CKD patients had comparatively higher short term and long term mortality if they developed CIAKI ${ }^{11}$. In yet another study, $12.7 \%$ of patients developed CIAKI after expose to contrast media and has a higher mortality 22 . Both of these studies stressed the relevance of contrast induced nephropathy in non-CRF patients in addition to the observed association of long-term mortality with small improvements in creatinine levels after acute coronary event, independent of the baseline eGFR ${ }^{21}$. In addition, patients whose serum creatinine returns to baseline after CIAKI, has been observed to have a continued risk of developing adverse effects compared to control ${ }^{21}$. These results have led to the understanding that certain therapeutic steps should be taken such as maintaining the volume status and avoidance of nephrotoxic drugs etc should be taken to slow the possible progression of CKD and prevent hospital read missions after an episode of AKI.

\section{CONCLUSION}

Acute kidney injury after coronary angiography is not uncommon in our population. More studies are recommended on its risk factors and complications to draw future directions for its control and prevention.

\section{CONFLICT OF INTEREST}

This study has no conflict of interest to be declared by any author.

\section{REFERENCES}

1. Pistolesi V, Regolisti G, Morabito S, Gandolfini I, Corrado S, Piotti G, Fiaccadori E.Contrast medium induced acute kidney injury: a narrative review. J Nephrol 2018; 31(6): 797-812.
2. Brown JR, Rezaee ME, Nichols EL, Marshall EJ, Siew ED, Matheny ME. Incidence and In-Hospital Mortality of Acute Kidney Injury (AKI) and Dialysis-Requiring AKI (AKI-D) After Cardiac Catheterization in the National Inpatient Sample. J Am Heart Assoc 2016; 5(1): e002739.

3. Moura ELB, Amorim FF, Huang W, Maia MO. Contrast-induced acute kidney injury: the importance of diagnostic criteria for establishing prevalence and prognosis in the intensive care unit. Rev Bras TerIntensiva 2017; 29(3): 303-09.

4. McDonald JS, McDonald RJ, Tran CL, Kolbe AB, Williamson EE, Kallmes DF. Postcontrast acute kidney injury in pediatric patients: a cohort study. Am J Kidney Dis 2018; 72(6): 811-18.

5. Molen AJV, Peter R, Ilona AD,Bongartz G, Bellin MF, Bertolotto $\mathrm{M}$, et al. Post-contrast acute kidney injury - Part 1: Definition, clinical features, incidence, role of contrast medium and risk factors. Recommendations for updated ESUR Contrast Medium Safety Committee guidelines. Eur Radiol 2018; 28(7): 2845-55.

6. Sandeep K, Ranjith KN, Naveen A, Abbot AK, Muthukrishnan J, Kumar HVS. Risk Factors For Contrast-Induced Nephropathy After Coronary Angiography. Saudi J Kidney Dis Transpl 2017; 28(2): 318-24.

7. Amini S, Najafi MN, Karrari SP, Mashhadi ME, Mirzaei S, Tashnizi MA, et al. Risk Factors and Outcome of Acute Kidney Injury after Isolated CABG Surgery: A Prospective Cohort Study. Braz J Cardiovasc Surg 2019; 34(1): 70-75.

8. HKim K, Joung KW, Ji SM, Kim JY, Lee EH, Chung $\mathrm{CH}$, et al. The effect of coronary angiography timing and use of cardiopulmonary bypass on acute kidney injury after coronary artery bypass graft surgery. J Thorac Cardiovasc Surg 2016; 152(1): 254-61.

9. Sercin O, Abdullah O. Contrast-induced Acute Kidney Injury: A review of practical points. World J Nephrol 2017; 6(3): 86-99.

10. Baek SD, Kim SM, Kang JY, Han M, lee KE, Jai WC. A Risk Scoring Model To Predict Renal Progression Associated With Postcontrast Acute Kidney Injury In Chronic Kidney Disease Patients. Medicine (Baltimore) 2019; 98(5): e14377.

11. Neyra JA, Shah S, Mooney R, Jacobsen G, Yee J, Novak JE. Contrast-induced Acute Kidney Injury Following Coronary Angiography: A Cohort Study Of Hospitalized Patients With Or Without Chronic Kidney Disease. Nephrol Dial Transplant 2013; 28(6): 1463-71.

12. Bhandari P, Shah Z, Patel K, Patel R. Contrast-induced acute kidney injury following coronary angiography in patients with end-stage liver disease. J Community Hosp Intern Med Perspect 2019; 9(5): 403-09.

13. Bruek M, Cengiz H, Hoeltgen R. Usefulness of N-acetylcysteine or ascorbic acid vs placebo to prevent contrast induced acute kidney injury in patients undergoing elective cardiac catheterization: a single center, prospective, double blind, placebo controlled trial. J Invasive Cardiol 2015; 25(1): 276-83.

14. Ribitsch W, Horina JH, Quehenberger F, Rosenkranz AR, Schilcher G. Contrast Induced Acute Kidney Injury and Its Impact on Mid-Term Kidney Function, Cardiovascular Events and Mortality. Sci Rep 2019; 9(1): 16896.

15. Weisbord SD, Chen H, Stone RA, Kip KE, Fine MJ, Saul MI. Associations of Increases In Serum Creatinine With Mortality And Length of Hospital Stay After Coronary Angiography. J Am Soc Nephrol 2006; 17(1): 2871-77.

16. McCullough PA, Adam A, Becker CR, Davidson C, Lameire N, Stacul F. CIN Consensus Working Panel. Risk Prediction of Contrast-Induced Nephropathy. Am J Cardiol 2006; 98(1): $27 \mathrm{~K}-36 \mathrm{~K}$.

17. Atanda AC, Olafiranye O. Contrast-induced acute kidney injury in interventional cardiology: emerging evidence and unifying 
mechanisms of protection by remote ischemic conditioning. Cardiovasc Revasc Med 2017; 18(7): 549-53.

18. Coca SG, Peixoto A, Garg AX, Krumholz H, Parikh CR. The prognostic importance of a small acute decrement in kidney function in hospitalized patients: a systematic review and metaanalysis. Am J Kidney Dis 2007; 50(5): 712-20.

19. Tsai TT, Patel UD, Chang TI, Kennedy KF, Masoudi FA, Matheny $\mathrm{ME}$, et al. Contemporary incidence, predictors, and outcomes of acute kidney injury in patients undergoing percutaneous coronary interventions: insights from the NCDR CathPCI registry. JACC Cardiovasc Interv 2014; 7(1): 1-9.

20. Pickering JW, James MT, Palmer SC. Acute Kidney Injury and Prognosis After Cardiopulmonary Bypass: A Meta-Analysis of Cohort Studies. Am J Kidney Dis 2015; 65(2): 283-93.

21. Dangas G, Iakovou I, Nikolsky E, Aymong ED, Mintz GS,
Kipshidze NN. Contrast-induced nephropathy after percutaneous coronary interventions in relation to chronic kidney disease and hemodynamic variables. Am J Cardiol 2005; 95(1): 13-19.

22. Wald R, Quinn RR, Luo J, Li P, Scales DC, Mamdani MM, Ray JG. Chronic dialysis and death among survivors of acute kidney injury requiring dialysis. J Am Med Assoc 2009; 302(11): 1179-85.

23. Shlipak MG, Katz R, Kestenbaum B, Shlipak MG, Katz R, Kestenbaum B. Rapid decline of kidney function increases cardiovascular risk in the elderly. J Am Soc Nephrol 2009; 20(12): 2625-30.

24. Greiner MA, Fonarow CG, Fonarow CG, Hammill BG. Relationship Between Early Physician Follow-Up And 30-Day Readmission Among Medicare Beneficiaries Hospitalized For Heart Failure. J Am Med Assoc 2010; 303(1): 1716-22. 